\section{Pesquisa sobre mortalidade perinatal no Brasil: revisão da metodologia e dos resultados}

\author{
Perinatal mortality research in Brazil: \\ review of methodology and results
}

\section{Abstract}

The perinatal mortality rate remains a public health problem, demanding epidemiological studies to describe its magnitude and time trends, identify risk factors, and define adequate interventions. There are still methodological controversies, resulting in heterogeneous studies and possible biases. In Brazil, there has been a growing scientific output on this theme, mainly in the South and Southeast of the country. Twentyfour articles from 1996 to 2003 were reviewed, focusing on definitions and classifications, data sources, study designs, measurement of variables, statistical analysis, and results. The review showed an increasing utilization of data bases (mainly SINASC and SIM), few studies on stillbirth, the incorporation of classification schemes, and disagreement concerning risk factors.

Perinatal Mortality; Review Literature; Methods
Sandra Costa Fonseca 1

Evandro da Silva Freire Coutinho 2

\section{Introdução}

A mortalidade perinatal, que engloba óbitos fetais e óbitos neonatais precoces, é um indicador de saúde materno-infantil, refletindo tanto as condições de saúde reprodutiva, por sua vez ligadas a fatores sócio-econômicos, quanto a qualidade da assistência perinatal - pré-natal, parto e neonatal 1 .

Anualmente ocorrem cerca de 7,6 milhões de mortes perinatais, das quais $98 \%$ nos países em desenvolvimento 2 . Nestes, cerca de $57 \%$ são representadas pelos óbitos fetais, cuja queda tem sido muito lenta. Comparada à natimortalidade, a neomortalidade tem apresentado maior redução, porém esta não é tão intensa quanto a que se apresenta nos países desenvolvidos, onde diminuiu-se a mortalidade em todas as faixas gestacionais e de peso $3,4,5$.

Por sua relevância em saúde pública, a mortalidade perinatal é objeto de vários estudos epidemiológicos. Observa-se, no entanto, grande heterogeneidade nos aspectos conceituais e metodológicos, tais como: definição, classificação, fontes de dados, delineamentos, determinantes, aferição de variáveis e análise estatística.

A definição atual 2 da mortalidade perinatal tem como numerador dois componentes - óbitos fetais a partir da 22a semana (natimortalidade) e os óbitos neonatais menores que sete dias de vida (neomortalidade precoce) - e como denominador o número total de nascimen- 
tos (vivos e mortos). Contudo, outras definições são usadas e não há homogeneidade para definir nascido vivo e natimorto 6,7,8. Para a definição de natimorto, são usados dois critérios: idade gestacional (28 semanas ou 24 semanas) e/ou peso - a partir de $500 \mathrm{~g}{ }^{8}$. É importante destacar que a natimortalidade, embora represente um dos desfechos desfavoráveis em saúde materno-infantil, é menos estudada que a neomortalidade, talvez pela insuficiência de registros 9,10. Quanto ao óbito neonatal, a maioria dos estudos considera apenas aqueles com menos de sete dias, havendo, no entanto, autores que advogam a inclusão de todos os óbitos neonatais (menos de 28 dias) no cálculo da mortalidade perinatal 6 . Recentemente, Kramer et al. 11 advogaram a extinção do termo mortalidade perinatal, já que, pelo menos nos países desenvolvidos, os determinantes da natimortalidade vêm-se distinguindo cada vez mais dos da neomortalidade.

As classificações de causas de mortalidade permitem sistematizar e compreender melhor a complexa determinação da mortalidade perinatal 12,13. Uma das mais conhecidas e utilizadas é a de Wigglesworth 14 , que serviu de base para outros modelos, como a de Keeling et al. 12. Esta classificação relaciona cinco categorias fisiopatológicas (anteparto, malformações, prematuridade, asfixia, infecções específicas e outras) à possível falha na assistência perinatal. No Brasil, a Fundação SEADE 15 desenvolveu uma classificação que agrupa as causas básicas, segundo a redutibilidade e a intervenção perinatal associada. Na Europa, os países nórdicos têm produzido modelos mais complexos de classificação, incorporando a avaliação do crescimento intra-uterino 8,13. Lansky et al. 16 revisaram as diferentes classificações e seu uso nos estudos de mortalidade perinatal, com destaque para a avaliação de evitabilidade dos óbitos.

Quanto às fontes de dados, o mais comum é a utilização de dados secundários, provenientes dos sistemas de informações sobre nascimentos e óbitos ou de registros hospitalares. A vinculação dos sistemas, através de técnicas de linkage, permite o monitoramento temporal de vários desfechos perinatais e o estudo de diversos fatores de risco 17. Outra vantagem é a base populacional, em substituição aos dados hospitalares, por vezes enviesados. No Brasil, o SINASC (Sistema de Informações sobre Nascidos Vivos) foi implantado em 1990 e só mais recentemente começou a ser utilizado para pesquisa, em conjunto com o SIM (Sistema de Informações de Mortalidade). Alguns problemas dessas fontes de dados -subnotificação, falta de preenchimento de variáveis e/ou a discordân- cia na informação entre dois sistemas - já foram suficientemente demonstradas 18,19,20. Outro problema é a escolha das variáveis que constam dos bancos. Por exemplo, em outros países, tabagismo, peso e altura materna - fatores de risco perinatal - constam da declaração de nascimento, enquanto, no Brasil, esses dados não são coletados. O formato da variável também pode ser limitante: a informação sobre idade gestacional no SINASC e no SIM é coletada de forma agrupada (faixas de idade gestacional) e não de forma contínua (número de semanas), impedindo avaliação mais precisa da prematuridade e do crescimento intra-uterino. Portanto, se, por um lado, as bases são uma ferramenta bastante útil para monitorar tendências e sugerir possíveis fatores, por outro, oferecem limitações para investigar fatores de risco perinatais.

Em relação aos delineamentos, a maioria dos estudos de risco utiliza o estudo de coorte; isso se explica pela disponibilidade de bases de dados populacionais, principalmente em países desenvolvidos, onde diversos fatores de risco são coletados de rotina. O estudo caso-controle é menos utilizado; contudo, mostra-se um delineamento adequado para estudos de causalidade, desde que vários requisitos metodológicos sejam atendidos, com especial destaque para a seleção dos controles 21,22.

O estudo da determinação de mortalidade perinatal está imbricado com o estudo do baixo peso ao nascer 23 e seus componentes - prematuridade e crescimento intra-uterino retardado (CIUR). Todavia, a mortalidade perinatal resulta de uma complexa cadeia causal, onde prematuridade e CIUR, com ou sem baixo peso, são os determinantes proximais, desencadeados, por sua vez, por fatores intermediários e distais. Como determinantes distais mais importantes, listam-se os fatores sócio-econômicos, que através da mediação de fatores intermediários, aumentam o risco de prematuridade e CIUR 24,25. Como fatores intermediários, listam-se: fatores biológicos maternos, história reprodutiva, hábitos maternos, doenças maternas, assistência pré e perinatal. Por conta da complexidade e da inter-relação entre os determinantes e da limitação de análises baseadas apenas em significância estatística, recomendam-se modelos de análise hierarquizada 24,26 .

Por fim, o processo de aferição das variáveis merece especial consideração. É comum que os estudos não especifiquem como foi a aferição ou usem diferentes instrumentos. Para idade gestacional (IG), é usada a data da última menstruação (DUM), a avaliação do tamanho 
do feto por exame ultra-sonográfico (USG) ou métodos de avaliação neonatal 27,28. Como bem estudado por Alexander et al. 29 , existe discordância entre estas estimativas de idade gestacional. Quanto à adequação de peso/IG (proxy do crescimento intra-uterino), também são usadas diferentes curvas, mas no Brasil, na maioria dos serviços, ainda se usa a de Lubchenco et al. 30 , considerada inadequada, tendendo a subestimar a prevalência de crianças pequenas para idade gestacional (PIG). Existem várias curvas mais recentes e/ou mais robustas do ponto de vista metodológico 31,32,33, 34,35,36. Em relação às demais variáveis estudadas na pesquisa sobre mortalidade perinatal, também se observa heterogeneidade quanto à sua aferição: diferentes conceitos e instrumentos são utilizados para mensurar nível sócioeconômico, pré-natal, tabagismo, alcoolismo, antropometria materna, morbidade e outros fatores.

Considerando todos esses aspectos metodológicos, este trabalho se propõe a rever a literatura recente sobre mortalidade perinatal, fetal e neonatal no Brasil.

\section{Metodologia}

Foi feita uma revisão bibliográfica (MEDLINE e LILACS) com as palavras-chave: mortalidade perinatal, fetal, ou neonatal; natimortalidade, neomortalidade, óbito fetal, óbito neonatal. Foram selecionados artigos originais, nos quais a mortalidade perinatal, fetal ou neonatal eram os desfechos principais em localidades brasileiras. O período de publicação selecionado foi 1996 a 2003, para refletir a produção mais recente sobre o tema (da década de 90 até a atualidade). Foram considerados artigos descritivos aqueles que apresentaram características e/ou classificações dos óbitos perinatais, descrevendo taxas ou séries temporais. Foram considerados artigos sobre determinação aqueles que exploraram a relação entre fatores de risco e os desfechos de mortalidade perinatal, fetal ou neonatal, estimando medidas de efeito. Identificaram-se e analisaram-se 24 artigos quanto aos aspectos metodológicos descritos anteriormente. A Tabela 1 apresenta as taxas de mortalidade encontradas nos diversos trabalhos e a Tabela 2, as estimativas de efeito dos determinantes de mortalidade perinatal, fetal e neonatal.
Tabela 1

Taxas de mortalidade perinatal, fetal e neonatal no Brasil na década de 90 (por mil nascimentos).

\begin{tabular}{|c|c|c|c|c|}
\hline & Perinatal & Fetal & $\begin{array}{l}\text { Neonatal } \\
\text { precoce }\end{array}$ & $\begin{array}{c}\text { Neonatal } \\
\text { total }\end{array}$ \\
\hline Brasil* & & 14,0 & 8,0 & 11,0 \\
\hline Norte* & & 11,0 & 7,0 & 8,0 \\
\hline Nordeste* & & 14,0 & 7,0 & 9,0 \\
\hline São Luís & & & & 28,2 \\
\hline Fortaleza & 32,8 & 17,9 & 15,1 & \\
\hline Centro-Oeste* & & 13,0 & 8,0 & 10,0 \\
\hline Goiânia & & & & 11,0 \\
\hline Sudeste* & & 16,0 & 11,0 & 13,0 \\
\hline Estado do Rio de Janeiro & & & & 17,4 \\
\hline Rio de Janeiro & 16,0 & & & \\
\hline Belo Horizonte & 20,2 & 9,0 & 11,2 & \\
\hline São Paulo & & & & 11,9 \\
\hline Campinas & & & & 11,0 \\
\hline Ribeirão Preto & & $8,9 * \star$ & & \\
\hline Sul* & & 12,0 & 8,0 & 11,0 \\
\hline Pelotas & 22,1 ** & $10,5^{\star \star}$ & 11,7 & 14,3 \\
\hline Caxias do Sul & & 13,7 & 7,4 & 12,7 \\
\hline
\end{tabular}

* Dados do artigo de Schramm \& Szwarcwald 62. Os demais resultados foram retirados dos artigos correspondentes às localidades, sendo considerados apenas aqueles com dados populacionais.

** Considerando apenas fetos com mais de 28 semanas

\section{Resultados}

\section{Estudos descritivos (Tabela 3)}

a) Região Sul: em Pelotas, Menezes et al. 37 compararam as taxas de mortalidade perinatal, fetal e neonatal precoce, referentes às coortes de 1982 e de 1993, abrangendo toda a população de nascimentos. Houve redução de $31 \%$ da mortalidade perinatal no período. O ponto de corte para os óbitos fetais foi de 28 semanas. A idade gestacional foi calculada pela DUM e o crescimento intra-uterino, pela curva de Williams et al. 31. Os óbitos foram descritos por sexo, peso, IG e CIUR, além de variáveis sócio-demográficas. Nas duas coortes, predominou a baixa idade gestacional, embora houvesse muitos casos com IG desconhecida. As causas básicas foram determinadas pelos pesquisadores, após análise de cada caso. Foi adotada a classificação de Wigglesworth (modificada por Keeling), mostrando que as mortes fetais anteparto e por imaturidade diminuíram e que as mal- 
Tabela 2

Estimativas de efeito dos principais determinantes de mortalidade.

\begin{tabular}{|c|c|c|c|c|}
\hline Determinante & Óbito perinatal & Óbito fetal & $\begin{array}{l}\text { Óbito neonatal } \\
\text { precoce }\end{array}$ & Óbito neonatal \\
\hline Hospital público/SUS & & & & $\begin{array}{l}2,358 \\
1,555\end{array}$ \\
\hline Assistência inadequada & & & $\Uparrow 51$ & $\Uparrow 59$ \\
\hline Adolescência & & $\begin{array}{l}0,3660 \\
2,053 \text { (NS) }\end{array}$ & $\begin{array}{c}4,149 \text { (NS) } \\
\Uparrow 54\end{array}$ & 1,555 \\
\hline$\geq 35$ anos & 2,448 & $\begin{array}{l}2,160 \text { (NS) } \\
2,548 \\
2,950\end{array}$ & $\begin{array}{l}2,7548 \\
4,5249\end{array}$ & NS 55 \\
\hline Peso $<50 \mathrm{~kg}$ & 1,448 (NS) & 1,148 (NS) & $\begin{array}{l}1,349 \text { (NS) } \\
1,648 \text { (NS) }\end{array}$ & \\
\hline Baixa renda & $\begin{array}{l}1,348 \text { (NS) } \\
4,356\end{array}$ & $\begin{array}{l}1,353 \text { (NS) } \\
2,160\end{array}$ & $\begin{array}{c}\Uparrow 54 \\
2,448 \text { (NS) }\end{array}$ & \\
\hline Residência precária & & & $\Uparrow 54$ & NS 55 \\
\hline Analfabetismo & & 3,360 & 4,649 (NS) & \\
\hline Baixa escolaridade & $\begin{array}{l}2,948 \\
2,856\end{array}$ & 3,048 & $\begin{array}{l}2,349 \text { (NS) } \\
1,648 \text { (NS) }\end{array}$ & NS 55 \\
\hline Tabagismo & 0,848 (NS) & 1,460 (NS) & 0,648 (NS) & \\
\hline Consumo de álcool & 0,948 (NS) & 1,360 (NS) & 0,948 (NS) & \\
\hline Morbidade materna & $\begin{array}{l}1,7560 \\
2,953\end{array}$ & & & \\
\hline Pré-natal inadequado & NS 49 & $\begin{array}{l}3,360 \\
7,453 \text { (NS) }\end{array}$ & $\begin{array}{l}3,148 \\
1,849 \text { (NS) }\end{array}$ & NS 55 \\
\hline № de partos $\geq 4$ & 0,948 (NS) & 0,948 (NS) & $\begin{array}{l}2,349 \text { (NS) } \\
1,353 \text { (NS) }\end{array}$ & \\
\hline Natimorto prévio & 2,548 (NS) & $\begin{array}{l}4,048 \\
5,253\end{array}$ & $\begin{array}{l}18,249 \\
2,648 \text { (NS) }\end{array}$ & \\
\hline Baixo peso prévio & 1,9848 & 2,948 & 1,648 (NS) & \\
\hline Sexo masculino & 1,5448 & $\begin{array}{l}1,248 \text { (NS) } \\
1,0660 \text { (NS) }\end{array}$ & $\begin{array}{l}1,8548 \\
4,249\end{array}$ & NS 55 \\
\hline Baixo peso ao nascer & & & $\begin{array}{r}36,448 \\
5,949\end{array}$ & $\begin{array}{l}8,9258 \\
6,9655\end{array}$ \\
\hline Muito baixo peso & & & 58,849 & 125,455 \\
\hline \multicolumn{2}{|l|}{$\mid$ dade gestacional $<37$ semanas } & & 5,649 & $\begin{array}{l}8,9458 \\
3,7555\end{array}$ \\
\hline PIG & & & 1,549 (NS) & 8,352 \\
\hline Apgar $<7$ & & & 10,149 & \\
\hline
\end{tabular}

NS = estatisticamente não significativo. Os valores numéricos expressam odds ratio ou razão de taxas.

$\Uparrow=$ expressa aumento do risco em estudos ecológicos.

Referências: 48. Menezes et al.; 49. Araújo et al.; 50. Vardanega et al.; 51. Leal \& Szwarcwald; 52. Almeida \& Mello

Jorge; 53. Aquino et al.; 54. Andrade \& Szwarcwald; 55. Almeida et al.; 56. Andrade et al.; 58. Morais Neto \& Barros

59. Morais Neto et al.; 60. Rouquayrol et al. 
Artigos descritivos - autor e periódico, características metodológicas.

\begin{tabular}{|c|c|c|c|c|c|c|c|c|}
\hline Autor & $\begin{array}{l}\text { Local do } \\
\text { estudo }\end{array}$ & Periódico & $\begin{array}{l}\text { Ano de } \\
\text { publicação }\end{array}$ & $\begin{array}{l}\text { Desfecho } \\
\text { estudado }\end{array}$ & $\begin{array}{l}\text { Definição } \\
\text { adotada }\end{array}$ & $\begin{array}{l}\text { Classificação } \\
\text { de causas }\end{array}$ & Metodologia & $\begin{array}{l}\text { Tipo de dados/ } \\
\text { período }\end{array}$ \\
\hline $\begin{array}{l}\text { Menezes } \\
\text { et al. } 37\end{array}$ & $\begin{array}{l}\text { Pelotas, Rio } \\
\text { Grande do Sul }\end{array}$ & $\begin{array}{l}\text { Cad Saúde } \\
\text { Pública }\end{array}$ & 1996 & $\begin{array}{l}\text { Mortalidade } \\
\text { perinatal, } \\
\text { fetal e neonatal } \\
\text { precoce }\end{array}$ & $\begin{array}{l}\text { Óbitos fetais } \\
\geq 28 \text { semanas } \\
\text { e neonatais } \\
<7 \text { dias } \\
\text { Peso } \geq 500 \mathrm{~g}\end{array}$ & $\begin{array}{l}\text { Wigglesworth } \\
\text { (adaptada p/ } \\
\text { Keeling) }\end{array}$ & $\begin{array}{l}\text { Base populacional } \\
\text { Coorte } 1982(n=194) \\
\text { vs. } 1993 \text { ( } n=117) \\
\text { Comparação temporal } \\
\text { de coeficientes (por } \\
\text { sexo, peso, ClUR } \\
\text { e grupo de causas) }\end{array}$ & $\begin{array}{l}\text { Primários } \\
1982 \text { e } 1993\end{array}$ \\
\hline $\begin{array}{l}\text { Leal \& } \\
\text { Szwarcwald } 3\end{array}$ & $\begin{array}{l}\text { Estado do } \\
\text { Rio de Janeiro }\end{array}$ & $\begin{array}{l}\text { Rev Saúde } \\
\text { Pública }\end{array}$ & 1996 & $\begin{array}{l}\text { Mortalidade } \\
\text { neonatal }\end{array}$ & $\begin{array}{l}\text { Óbitos < } 28 \\
\text { dias }\end{array}$ & Nenhuma & $\begin{array}{l}\text { Base populacional } \\
\text { Série temporal } \\
\text { Variação anual (taxa) }\end{array}$ & $\begin{array}{l}\text { Secundários } \\
\text { SIM/IBGE } \\
1979 \text { a } 1993\end{array}$ \\
\hline $\begin{array}{l}\text { Leal \& } \\
\text { Szwarcwald } 40\end{array}$ & $\begin{array}{l}\text { Estado do } \\
\text { Rio de Janeiro }\end{array}$ & $\begin{array}{l}\text { Cad Saúde } \\
\text { Pública }\end{array}$ & 1996 & $\begin{array}{l}\text { Mortalidade } \\
\text { neonatal }\end{array}$ & $\begin{array}{l}\text { Óbitos < } 28 \\
\text { dias }\end{array}$ & $\begin{array}{l}\text { Fundação } \\
\text { SEADE }\end{array}$ & $\begin{array}{l}\text { Base populacional } \\
\text { Variação dos coefi- } \\
\text { cientes por causas } \\
\text { e da mortalidade } \\
\text { proporcional }\end{array}$ & $\begin{array}{l}\text { Secundários } \\
\text { SIM/IBGE } \\
1979 \text { a } 1993\end{array}$ \\
\hline $\begin{array}{l}\text { Leite } \\
\text { et al. } 46\end{array}$ & $\begin{array}{l}\text { Fortaleza, } \\
\text { Ceará }\end{array}$ & $\begin{array}{l}\text { J Pediatr } \\
\text { (Rio J) }\end{array}$ & 1997 & $\begin{array}{l}\text { Mortalidade } \\
\text { perinatal, } \\
\text { fetal e neonatal } \\
\text { precoce }\end{array}$ & $\begin{array}{l}\text { Óbitos fetais } \\
\text { e neonatais } \\
\text { precoces } \mathrm{c} / \\
\text { peso } \geq 500 \mathrm{~g}\end{array}$ & $\begin{array}{l}\text { Wigglesworth } \\
\text { (Keeling) }\end{array}$ & $\begin{array}{l}\text { Base populacional } \\
(n=1.337) \\
\text { Coorte } \\
\text { Coeficientes por peso } \\
\text { e grupo de causas }\end{array}$ & $\begin{array}{l}\text { Secundários } \\
\text { SINASC } \\
\text { Prontuários } \\
\text { DO } \\
1995\end{array}$ \\
\hline $\begin{array}{l}\text { Miura } \\
\text { et al. } 38\end{array}$ & $\begin{array}{l}\text { Porto Alegre, } \\
\text { Rio Grande } \\
\text { do Sul }\end{array}$ & $\begin{array}{l}\text { Rev Assoc } \\
\text { Med Bras }\end{array}$ & 1997 & $\begin{array}{l}\text { Mortalidade } \\
\text { perinatal, } \\
\text { fetal e neonatal }\end{array}$ & $\begin{array}{l}\text { Óbitos fetais } \\
\text { e neonatais } \\
\text { Peso } \geq 500 \mathrm{~g}\end{array}$ & Quebec & $\begin{array}{l}\text { Base hospitalar } \\
\text { ( } \mathrm{n}=632 \text { ) } \\
\text { Comparação de } \\
\text { causas básicas } \\
\text { Taxas por faixa } \\
\text { de peso }\end{array}$ & $\begin{array}{l}\text { Secundários } \\
\text { Prontuários } \\
1984-1990\end{array}$ \\
\hline $\begin{array}{l}\text { Sardas \& } \\
\text { Manco } 41\end{array}$ & $\begin{array}{l}\text { Ribeirão Preto, } \\
\text { São Paulo }\end{array}$ & $\begin{array}{l}\text { Medicina } \\
\text { (Ribeirão } \\
\text { Preto) }\end{array}$ & 1997 & $\begin{array}{l}\text { Mortalidade } \\
\text { fetal }\end{array}$ & $\begin{array}{l}\text { Óbitos fetais } \\
>20 \text { semanas }\end{array}$ & CID-9 & $\begin{array}{l}\text { Base populacional } \\
\text { ( } n=299) \\
\text { Descrição por peso, } \\
\text { IG e causas básicas }\end{array}$ & $\begin{array}{l}\text { Secundários } \\
\text { D. O. } \\
1991-1992\end{array}$ \\
\hline $\begin{array}{l}\text { Aquino \& } \\
\text { Cecatti } 43\end{array}$ & São Paulo & $\begin{array}{l}\text { Rev Bras } \\
\text { Ginecol } \\
\text { Obstet }\end{array}$ & 1998 & $\begin{array}{l}\text { Mortalidade } \\
\text { fetal }\end{array}$ & $\begin{array}{l}\text { Óbitos fetais } \\
\text { (anteparto) } \\
>20 \text { semanas }\end{array}$ & Nenhuma & $\begin{array}{l}\text { Base hospitalar } \\
(\mathrm{n}=122) \\
\text { Descrição por causas }\end{array}$ & $\begin{array}{l}\text { Primários } \\
\text { 1996-1997 }\end{array}$ \\
\hline $\begin{array}{l}\text { De Lorenzi } \\
\text { et al. } 39\end{array}$ & $\begin{array}{l}\text { Caxias do Sul, } \\
\text { Rio Grande } \\
\text { do Sul }\end{array}$ & $\begin{array}{l}\text { Rev Cient } \\
\text { AMECS }\end{array}$ & 1999 & $\begin{array}{l}\text { Mortalidade } \\
\text { fetal }\end{array}$ & $\begin{array}{l}\text { Óbitos fetais } \\
\geq 22 \text { semanas } \\
\text { Peso }>500 \mathrm{~g}\end{array}$ & Nenhuma & $\begin{array}{l}\text { Base populacional } \\
(n=78) \\
\text { Descrição dos } \\
\text { percentuais por causa, } \\
\text { peso e IG }\end{array}$ & $\begin{array}{l}\text { Secundários } \\
\text { D.O./Prontuário } \\
1996 \text { e } 1997\end{array}$ \\
\hline $\begin{array}{l}\text { Ribeiro \& } \\
\text { Silva } 47\end{array}$ & $\begin{array}{l}\text { São Luís, } \\
\text { Maranhão }\end{array}$ & $\begin{array}{l}\text { Cad Saúde } \\
\text { Pública }\end{array}$ & 2000 & $\begin{array}{l}\text { Mortalidade } \\
\text { neonatal }\end{array}$ & $\begin{array}{l}\text { Óbitos }<28 \\
\text { dias }\end{array}$ & $\begin{array}{l}\text { Fundação } \\
\text { SEADE }\end{array}$ & $\begin{array}{l}\text { Base populacional } \\
\text { ( } n=5.435 \text { ) } \\
\text { Série temporal } \\
\text { Variação do coeficiente }\end{array}$ & $\begin{array}{l}\text { Secundários } \\
\text { SIM/ IBGE } \\
\text { 1979-1996 }\end{array}$ \\
\hline $\begin{array}{l}\text { Schramm \& } \\
\text { Swarcwald } 62\end{array}$ & Brasil & $\begin{array}{l}\text { Cad Saúde } \\
\text { Pública }\end{array}$ & 2000 & $\begin{array}{l}\text { Mortalidade } \\
\text { fetal, neonatal } \\
\text { precoce e tardia }\end{array}$ & $\begin{array}{l}\text { Óbitos fetais } \\
\text { de qualquer } \\
\text { IG/peso } \\
\text { neonatal }<7 \\
\text { e } 7-27 \text { dias }\end{array}$ & Nenhuma & $\begin{array}{l}\text { Base populacional } \\
\text { Comparação de taxas }\end{array}$ & $\begin{array}{l}\text { Secundários } \\
\text { SIH/SUS } \\
1995\end{array}$ \\
\hline $\begin{array}{l}\text { Mariotoni \& } \\
\text { Barros Filho } 44\end{array}$ & $\begin{array}{l}\text { Campinas, } \\
\text { São Paulo }\end{array}$ & $\begin{array}{l}\text { Rev Saúde } \\
\text { Pública }\end{array}$ & 2000 & $\begin{array}{l}\text { Mortalidade } \\
\text { neonatal } \\
\text { hospitalar }\end{array}$ & $\begin{array}{l}\text { Óbitos } \\
\text { hospitalares } \\
\text { mesmo que } \\
>28 \text { dias }\end{array}$ & Nenhuma & $\begin{array}{l}\text { Base hospitalar } \\
(n=1.134) \\
\text { Coeficientes por } \\
\text { faixa de peso }\end{array}$ & $\begin{array}{l}\text { Secundários } \\
\text { Registros } \\
\text { hospitalares } \\
\text { 1975-1996 }\end{array}$ \\
\hline $\begin{array}{l}\text { Lansky } \\
\text { et al. } 45\end{array}$ & $\begin{array}{l}\text { Belo Horizonte, } \\
\text { Minas Gerais }\end{array}$ & $\begin{array}{l}\text { Cad Saúde } \\
\text { Pública }\end{array}$ & 2002 & $\begin{array}{l}\text { Mortalidade } \\
\text { perinatal, fetal } \\
\text { e neonatal } \\
\text { precoce }\end{array}$ & $\begin{array}{l}\text { Óbitos fetais } \\
>22 \text { semanas } \\
\text { e/ou }>500 \mathrm{~g} \\
\text { Óbitos neonatais } \\
<7 \text { dias e }>500 \mathrm{~g}\end{array}$ & $\begin{array}{l}\text { Wigglesworth } \\
\text { (Keeling) } \\
\text { s }\end{array}$ & $\begin{array}{l}\text { Base populacional } \\
(\mathrm{n}=826) \\
\text { Coorte } \\
\text { Coeficientes por peso } \\
\text { e grupo de causas }\end{array}$ & $\begin{array}{l}\text { Secundários } \\
\text { SINASC/D.O. } \\
\text { Prontuários } \\
1999\end{array}$ \\
\hline
\end{tabular}

$\mathrm{n}$ = número total de óbitos analisados considerado-se a base de estudo. Em alguns trabalhos, este valor não foi especificado.

$\mathrm{SIM}=$ Sistema de Informação de Mortalidade; SINASC = Sistema de Informações sobre Nascidos Vivos; IBGE = Fundação Instituto

Brasileiro de Geografia e Estatística; DO = Declaração de óbito; CIUR = Crescimento intra-uterino retardado; IG = Idade gestacional. 
formações praticamente não se alteraram; por outro lado, houve aumento dos óbitos por asfixia. Esses resultados sugerem melhoria das condições maternas e de assistência pré-natal e neonatal, ao passo que apontam problemas no cuidado obstétrico.

Miura et al. 38 apresentaram as características da mortalidade perinatal, fetal e neonatal (óbitos $\geq 500 \mathrm{~g}$ ), obtidas dos registros e prontuários do Hospital de Clínicas de Porto Alegre, de 1984-1990. Descreveram os coeficientes para o período completo, e não a evolução temporal. A taxa de mortalidade perinatal foi $28,4 \%$; a fetal, 18,4\%o; a neonatal, 12,7\%o. Não houve avaliação por idade gestacional ou crescimento intra-uterino, apenas por faixa de peso. As causas básicas foram comparadas em dois períodos: 1984-1987 e 1988-1990. Foi utilizada a classificação do Quebec 38. Para os neomortos, as causas mais comuns foram infecção intra-uterina e doença da membrana hialina, com aumento significativo de óbitos por malformações no segundo período; para os natimortos, foram asfixia e infecções intra-uterinas e toxemia, com poucas diferenças entre os dois períodos.

Em Caxias do Sul, Rio Grande do Sul, De Lorenzi et al. 39 avaliaram a natimortalidade óbitos de maiores que $500 \mathrm{~g}$ e com 22 semanas ou mais - no período de 1996 a 1997, tendo como base declarações de óbito (DO), revisão de prontuários e entrevistas. Foram calculados os percentuais por faixa de peso, IG e causas. Dois terços dos casos tinham peso menor que $2.500 \mathrm{~g}$ e idade gestacional menor que 37 semanas. Não é explicitado o critério de definição de IG. Apenas $6 \%$ foram classificados como PIG (usando a referência de Lubchenco ${ }^{30}$ ). A população se restringiu a pacientes do SUS e a causa básica mais comum foi hipertensão.

b) Região Sudeste: Leal \& Szwarcwald 3,40 descreveram a evolução da mortalidade neonatal total (menos de 28 dias) no Estado do Rio de Janeiro, de 1979 a 1993, usando dados populacionais do SIM e do IBGE (Fundação Instituto Brasileiro de Geografia e Estatística). A evolução temporal foi analisada considerandose a variação anual, calculada por regressão exponencial. No primeiro artigo, são analisadas três grandes áreas do estado: capital, cinturão metropolitano e interior. A mortalidade neonatal teve decréscimo lento em todas as áreas, sendo o pior desempenho no interior. Na análise por faixa etária (número de dias de vida), observou-se que quanto mais perto do nascimento, menor a queda da mortalidade; nas primeiras horas de vida, chegou a ocorrer aumento do coeficiente. Não houve análise por peso e idade gestacional. No segundo artigo, as auto- ras utilizaram a classificação da Fundação SEADE ampliada, para analisar a evolução de causas de óbito. Foram calculados os coeficientes por causa e a mortalidade proporcional. Destacouse a baixa qualidade da informação para as causas básicas, principalmente na primeira semana de vida, dificultando a interpretação dos resultados. Considerando essa limitação, houve diminuição de óbitos por imaturidade e CIUR no período estudado, enquanto aumentaram os óbitos por problemas respiratórios, e aqueles por asfixia se mantiveram inalterados.

Em Ribeirão Preto, São Paulo, Sardas \& Manço 41 relataram os óbitos fetais - considerados a partir de vinte semanas - no período de 1991 a 1992, tendo como fonte de informação as declarações de óbito registradas na cidade $(40 \%$ provenientes de outros municípios). Os óbitos foram descritos segundo faixa de peso, IG e idade materna, assinalando-se a ausência desses dados em mais de 30,0\% dos atestados. Dos casos com peso e IG conhecidos, $73,0 \%$ eram de fetos abaixo de $2.500 \mathrm{~g}$ e $73,0 \%$ tinham 28 ou mais semanas. As causas mais freqüentes, de acordo com a CID-9 42, foram hipóxia intra-uterina e complicações de placenta, cordão e membranas.

Aquino \& Cecatti 43 realizaram análise descritiva dos óbitos fetais com mais de vinte semanas, ocorridos antes do trabalho de parto, em unidade hospitalar de São Paulo, entre 1996 e 1997. O estudo foi prospectivo e os resultados foram apresentados na forma de média e desvio padrão para variáveis como peso, IG, idade materna e consultas de pré-natal. Não há descrição da taxa de mortalidade fetal. Do total de óbitos, 25,0\% foram por causas indeterminadas e 20,0\% relacionados à doença hipertensiva.

Mariotoni \& Barros Filho 44 descreveram as taxas de mortalidade hospitalar por peso ao nascer, em uma maternidade responsável por metade dos nascimentos de Campinas, São Paulo. Foram usados registros hospitalares durante o período de 1975 a 1996. Não houve ponto de corte para definir mortalidade neonatal; em alguns casos, de número não especificado, o óbito pode ter ocorrido após 28 dias de vida. Não há descrição de IG. A mortalidade neonatal hospitalar diminuiu entre 1975 e 1996, apesar da distribuição por faixas de peso ter se mantido constante em todo o período.

Lansky et al. 45 estudaram 826 óbitos perinatais ocorridos no ano de 1999, em Belo Horizonte, Minas Gerais, e analisaram a possibilidade de prevenção, valendo-se da classificação de Wigglesworth modificada. Foram estudados os nascidos mortos com peso acima de $500 \mathrm{~g}$ e/ ou idade gestacional maior que 22 semanas e óbitos neonatais precoces com peso ao nas- 
cer acima de 500g. As informações sobre os nascidos vivos foram obtidas do SINASC/Belo Horizonte, cuja cobertura chega a $100,0 \%$. Os óbitos foram avaliados com base na consulta às declarações e prontuários hospitalares, para confirmação da causa básica e classificação. Os coeficientes foram apresentados por faixas de peso e classificação de causa. Predominou o grupo de asfixia intraparto $(34,0 \%)$, seguido dos óbitos anteparto $(30,0 \%)$ e por imaturidade $(22,6 \%)$.

c) Região Nordeste: Leite et al. 46 analisaram a mortalidade perinatal e neonatal, estimando as taxas e a proporção de mortes evitáveis. $\mathrm{O}$ critério para definir nascido vivo e natimorto foi o peso igual ou superior a $500 \mathrm{~g}$. Foram acompanhados da coorte de 1995 todos os nascimentos nas maternidades públicas e conveniadas (SUS/Fortaleza), responsáveis por $85 \%$ dos partos na cidade. A informação foi retirada de prontuários, registros hospitalares, declarações de óbitos e do SINASC. Não foram avaliados IG ou CIUR. Os óbitos foram classificados por peso (apenas 0,08\% sem informação) e grupo de causas (Wigglesworth modificado por Keeling) para avaliar sua evitabilidade. De acordo com a classificação utilizada, $48,4 \%$ foram óbitos fetais anteparto; $34,7 \%$ por imaturidade; $8,9 \%$ por asfixia; $5,4 \%$ por malformações e o restante por condições específicas. Foram considerados evitáveis $34,0 \%$ dos óbitos perinatais, apontando para problemas na qualidade do pré-natal e da assistência neonatal terciária.

Ribeiro \& Silva 47 acompanharam a evolução da mortalidade neonatal - menos de 28 dias - em São Luís, Maranhão, nos anos de 1979 a 1996, usando como fonte de dados o SIM e o IBGE. Foi calculada a variação anual, por regressão exponencial. A mortalidade neonatal teve aumento importante no período estudado, principalmente a neomortalidade precoce, concentrada no primeiro dia de vida. Não houve descrição de peso ou IG. Em relação às causas, de acordo com a classificação da Fundação SEADE, ocorreu aumento das causas reduzíveis por diagnóstico precoce e das parcialmente reduzíveis pelo controle da gravidez. Este aumento pode ser explicado, em parte, por melhoria no registro de óbitos, mas certamente houve piora na assistência perinatal.

d) Brasil: Schramm \& Szwarcwald 20 utilizaram o Sistema de Informações Hospitalares (SIH) do SUS para estudar as taxas de mortalidade neonatal - precoce e tardia - e natimortalidade hospitalares no país no ano de 1995. Pelo tipo de fonte de dados, não foi avaliado peso, IG, CIUR ou causa básica. As grandes variações encontradas apontaram para subestimativas nas Regiões Norte e Nordeste.

\section{Estudos sobre determinação (Tabela 4)}

a) Região Sul: em Pelotas, Menezes et al. 48 acompanharam a coorte de nascimentos de 1993, estudando as causas de mortalidade perinatal, fetal (com 28 semanas ou mais) e neonatal precoce (menos de sete dias). A idade gestacional (baseada na DUM) não foi analisada por causa do grande sub-registro. Não houve distinção por grupo de causa básica. A análise estatística foi por regressão logística, segundo um modelo hierárquico, no qual fatores sócio-econômicos estavam no nível distal; fatores reprodutivos e biológicos, no nível intermediário, e o peso ao nascer, no nível proximal. Os determinantes encontrados para mortalidade perinatal foram: baixa escolaridade, idade materna elevada e sexo masculino. Para a neomortalidade precoce, esses fatores foram idade materna elevada, pré-natal inadequado (menos de cinco consultas), baixo peso do recémnascido ( $\mathrm{RN}$ ) e sexo masculino, enquanto para natimortalidade foram a baixa escolaridade materna (menor que quatro anos) e a idade da mãe igual ou superior a 35 anos.

Araújo et al. 49 identificaram os determinantes da mortalidade neonatal precoce (sete dias ou menos) em Caxias do Sul, na coorte de nascimentos hospitalares do ano de 1995. As crianças foram acompanhadas até sete dias de vida, e a mortalidade concentrou-se no primeiro dia de vida. Variáveis sócio-demográficas, reprodutivas e características do RN foram coletadas pelos pesquisadores. Não há referência ao método usado para estimar idade gestacional e crescimento intra-uterino. A análise estatística foi multivariada (regressão logística). Os fatores mais relevantes foram idade materna superior ou igual a 35 anos, história de natimorto anterior, Apgar $<7$, prematuridade, baixo peso e sexo masculino.

Também em Caxias do Sul, Vardanega et al. 50 estudaram os fatores materno-fetais e assistência pré-natal para a ocorrência de óbito fetal com 22 semanas ou mais e/ou peso superior ou igual a $500 \mathrm{~g}$, em um estudo caso-controle retrospectivo. Os dados foram retirados dos prontuários de uma unidade hospitalar, incluindo somente os casos ocorridos antes do trabalho de parto. O pré-natal foi avaliado qualitativamente, usando critérios de adequação do Ministério da Saúde. Apenas idade materna elevada (acima de 35 anos) foi considerada determinante do óbito fetal após análise multivariada.

b) Região Sudeste: Leal \& Szwarcwald 51 exploraram a dimensão espacial de indicadores sócio-ambientais e de assistência médica para 
Tabela 4

Artigos sobre determinação - autor, periódico, ano, características metodológicas.

\begin{tabular}{|c|c|c|c|c|c|c|c|c|}
\hline Autor & $\begin{array}{l}\text { Local do } \\
\text { estudo }\end{array}$ & Periódico & $\begin{array}{l}\text { Ano de } \\
\text { publicação }\end{array}$ & Desfecho & Definição & $\begin{array}{l}\text { Base } \\
\text { populacional } \\
\text { (no casos) } \\
\text { Desenho }\end{array}$ & $\begin{array}{l}\text { Tipo de dados/ } \\
\text { período }\end{array}$ & Análise \\
\hline $\begin{array}{l}\text { Rouquayrol } \\
\text { et al. } 60\end{array}$ & $\begin{array}{l}\text { Fortaleza, } \\
\text { Ceará }\end{array}$ & $\begin{array}{l}\text { J Pediatr } \\
\text { (Rio J) }\end{array}$ & 1996 & $\begin{array}{l}\text { Mortalidade } \\
\text { fetal }\end{array}$ & $\begin{array}{l}\text { Óbitos fetais } \\
>20 \text { semanas }\end{array}$ & $\begin{array}{l}\text { Base hospitalar } \\
(\mathrm{n}=125) \\
\text { Caso-controle }\end{array}$ & $\begin{array}{l}\text { Primários } \\
\text { 1992-1993 }\end{array}$ & $\begin{array}{l}\text { Multivariada } \\
\text { (hierarquizada) }\end{array}$ \\
\hline $\begin{array}{l}\text { Leal \& } \\
\text { Swarcwald } 51\end{array}$ & $\begin{array}{l}\text { Estado do } \\
\text { Rio de Janeiro }\end{array}$ & $\begin{array}{l}\text { Rev Saúde } \\
\text { Pública }\end{array}$ & 1997 & $\begin{array}{l}\text { Mortalidade } \\
\text { neonatal }\end{array}$ & $\begin{array}{l}\text { Óbitos }<28 \\
\text { dias }\end{array}$ & $\begin{array}{l}\text { Base populacional } \\
\text { Ecológico (geo- } \\
\text { processamento) }\end{array}$ & $\begin{array}{l}\text { Secundários } \\
\text { SIM/IBGE } \\
\text { 1979-1981 } \\
\text { e 1990-1992 }\end{array}$ & $\begin{array}{l}\text { Autocorrelação } \\
\text { espacial }\end{array}$ \\
\hline $\begin{array}{l}\text { Almeida \& } \\
\text { Mello-Jorge } 52\end{array}$ & $\begin{array}{l}\text { Santo André, } \\
\text { São Paulo }\end{array}$ & $\begin{array}{l}\text { Rev Saúde } \\
\text { Pública }\end{array}$ & 1998 & $\begin{array}{l}\text { Mortalidade } \\
\text { neonatal }\end{array}$ & $\begin{array}{l}\text { Óbitos }<28 \\
\text { dias }\end{array}$ & $\begin{array}{l}\text { Base populacional } \\
(n=55) \\
\text { Coorte }\end{array}$ & $\begin{array}{l}\text { Secundários } \\
\text { SINASC/SIM } \\
\text { Prontuários } \\
1992\end{array}$ & Bivariada \\
\hline $\begin{array}{l}\text { Menezes } \\
\text { et al. } 48\end{array}$ & $\begin{array}{l}\text { Pelotas, Rio } \\
\text { Grande do Sul }\end{array}$ & $\begin{array}{l}\text { Rev Saúde } \\
\text { Pública }\end{array}$ & 1998 & $\begin{array}{l}\text { Mortalidade } \\
\text { perinatal }\end{array}$ & $\begin{array}{l}\text { Óbitos fetais } \\
\geq 28 \text { semanas } \\
\text { Óbitos } \\
\text { neonatais } \\
<7 \text { dias }\end{array}$ & $\begin{array}{l}\text { Base populacional } \\
(\mathrm{n}=117) \\
\text { Coorte }\end{array}$ & $\begin{array}{l}\text { Primários } \\
1993\end{array}$ & $\begin{array}{l}\text { Multivariada } \\
\text { (hierarquizada) }\end{array}$ \\
\hline $\begin{array}{l}\text { Aquino } \\
\text { et al. } 53\end{array}$ & São Paulo & $\begin{array}{l}\text { São Paulo } \\
\text { Med J }\end{array}$ & 1998 & $\begin{array}{l}\text { Mortalidade } \\
\text { fetal }\end{array}$ & $\begin{array}{l}\text { Óbitos fetais } \\
\geq 20 \text { semanas } \\
\text { (anteparto) }\end{array}$ & $\begin{array}{l}\text { Base hospitalar } \\
(n=122) \\
\text { Caso-controle }\end{array}$ & $\begin{array}{l}\text { Primários } \\
\text { 1996-1997 }\end{array}$ & Multivariada \\
\hline $\begin{array}{l}\text { Morais Neto } \\
\& \text { Barros } 58\end{array}$ & Goiânia, Goiás & $\begin{array}{l}\text { Cad Saúde } \\
\text { Pública }\end{array}$ & 2000 & $\begin{array}{l}\text { Mortalidade } \\
\text { neonatal }\end{array}$ & $\begin{array}{l}\text { Óbitos }<28 \\
\text { dias }\end{array}$ & $\begin{array}{l}\text { Base populacional } \\
(n=231) \\
\text { Coorte }\end{array}$ & $\begin{array}{l}\text { Secundários } \\
\text { SINASC/SIM } \\
1992\end{array}$ & $\begin{array}{l}\text { Multivariada } \\
\text { (hierarquizada) }\end{array}$ \\
\hline $\begin{array}{l}\text { Morais Neto } \\
\text { et al. } 59\end{array}$ & Goiânia, Goiás & $\begin{array}{l}\text { Cad Saúde } \\
\text { Pública }\end{array}$ & 2001 & $\begin{array}{l}\text { Mortalidade } \\
\text { neonatal }\end{array}$ & $\begin{array}{l}\text { Óbitos }<28 \\
\text { dias }\end{array}$ & $\begin{array}{l}\text { Base populacional } \\
\text { Ecológico (geo- } \\
\text { processamento) }\end{array}$ & $\begin{array}{l}\text { Secundários } \\
\text { SINASC/SIM } \\
\text { 1992-1996 }\end{array}$ & $\begin{array}{l}\text { Autocorrelação } \\
\text { espacial }\end{array}$ \\
\hline $\begin{array}{l}\text { Araújo } \\
\text { et al. } 49\end{array}$ & $\begin{array}{l}\text { Caxias do Sul, } \\
\text { Rio Grande } \\
\text { do Sul }\end{array}$ & $\begin{array}{l}\text { J Pediatr } \\
\text { (Rio J) }\end{array}$ & 2000 & $\begin{array}{l}\text { Mortalidade } \\
\text { neonatal } \\
\text { precoce }\end{array}$ & $\begin{array}{l}\text { Óbitos } \\
\text { neonatais } \\
\leq 7 \text { dias }\end{array}$ & $\begin{array}{l}\text { Base populacional } \\
(n=42) \\
\text { Coorte }\end{array}$ & $\begin{array}{l}\text { Primários e } \\
\text { secundários } \\
\text { Prontuários } \\
1995\end{array}$ & Multivariada \\
\hline $\begin{array}{l}\text { Andrade \& } \\
\text { Swarcwald } 54\end{array}$ & Rio de Janeiro & $\begin{array}{l}\text { Cad Saúde } \\
\text { Pública }\end{array}$ & 2001 & $\begin{array}{l}\text { Mortalidade } \\
\text { neonatal do } \\
\text { 1o ao } 3 \text { o dia }\end{array}$ & $\begin{array}{l}\text { Óbitos } \\
\text { neonatais } \\
\leq 3 \text { dias }\end{array}$ & $\begin{array}{l}\text { Base populacional } \\
\text { Ecológico (geo- } \\
\text { processamento) }\end{array}$ & $\begin{array}{l}\text { Secundários } \\
\text { SINASC/SIM } \\
\text { Censo IBGE } \\
\text { 1995-1996 }\end{array}$ & $\begin{array}{l}\text { Autocorrelação } \\
\text { espacial }\end{array}$ \\
\hline $\begin{array}{l}\text { Almeida } \\
\text { et al. } 55\end{array}$ & São Paulo & $\begin{array}{l}\text { Rev Bras } \\
\text { Epidemiol }\end{array}$ & 2002 & $\begin{array}{l}\text { Mortalidade } \\
\text { neonatal }\end{array}$ & $\begin{array}{l}\text { Óbitos } \\
\text { neonatais }\end{array}$ & $\begin{array}{l}\text { Base populacional } \\
(\mathrm{n}=893) \\
\text { Caso-controle }\end{array}$ & $\begin{array}{l}\text { Secundários } \\
\text { SINASC/SIM } \\
1995\end{array}$ & $\begin{array}{l}\text { Multivariada } \\
\text { (hierarquizada) }\end{array}$ \\
\hline $\begin{array}{l}\text { Vardanega } \\
\text { et al. } 50\end{array}$ & $\begin{array}{l}\text { Caxias do Sul, } \\
\text { Rio Grande } \\
\text { do Sul }\end{array}$ & $\begin{array}{l}\text { Rev Bras } \\
\text { Ginecol } \\
\text { Obstet }\end{array}$ & 2002 & $\begin{array}{l}\text { Mortalidade } \\
\text { fetal }\end{array}$ & $\begin{array}{l}\text { Óbitos fetais } \\
\geq 22 \text { semanas } \\
\text { e/ou } \geq 500 \mathrm{~g} \\
\text { (anteparto) }\end{array}$ & $\begin{array}{l}\text { Base hospitalar } \\
(\mathrm{n}=60) \\
\text { Caso-controle } \\
\text { (retrospectivo) }\end{array}$ & $\begin{array}{l}\text { Secundários } \\
\text { Prontuários } \\
\text { 1998-2001 }\end{array}$ & Multivariada \\
\hline $\begin{array}{l}\text { Andrade } \\
\text { et al. } 56\end{array}$ & Rio de Janeiro & $\begin{array}{l}\text { Cad Saúde } \\
\text { Pública }\end{array}$ & 2004 & $\begin{array}{l}\text { Mortalidade } \\
\text { perinatal }\end{array}$ & $\begin{array}{l}\text { Óbito entre } \\
\text { 22a semana } \\
\text { de gestação e } \\
\text { o } 7 \underline{\text { o dia de vida }}\end{array}$ & $\begin{array}{l}\text { Base populacional } \\
\text { Transversal }\end{array}$ & $\begin{array}{l}\text { Primários } \\
\text { Secundários } \\
\text { Prontuários } \\
\text { 1999-2001 }\end{array}$ & Bivariada \\
\hline
\end{tabular}

$\mathrm{n}=$ número total de óbitos analisados considerando-se a base de estudo. Em alguns trabalhos, esse valor não foi especificado. $\mathrm{SIM}=$ Sistema de informação de mortalidade; SINASC = Sistema de informações sobre nascidos vivos; IBGE = Fundação Instituto Brasileiro de Geografia e Estatística. 
explicar a ocorrência de mortalidade neonatal no Estado do Rio de Janeiro, comparando as décadas de 80 e 90. As fontes de dados para nascimentos e óbitos foram respectivamente as estatísticas de registro civil e o SIM. Para os fatores de risco sócio-ambientais - escolaridade, renda, condições sanitárias -, foi construído um indicador composto, chamado nível sócio-econômico (NSE), com base nos censos demográficos (IBGE). Para assistência médica, foi usado número de leitos, número de obstetras e proporção de partos cesáreos (fonte: Assistência Médico Sanitária, IBGE). Neste estudo ecológico, foram utilizadas medidas de autocorrelação espacial na análise estatística. A mortalidade neonatal foi dividida em três grupos: primeiro dia de vida, um a seis dias e sete a 27 dias. Não foi usada classificação por causas nem distinção entre prematuridade e CIUR. Os resultados mostraram que, na década de 80 , havia associação entre os indicadores sócioeconômicos e a mortalidade; entretanto, na década de 90, destacou-se a relação entre os indicadores de assistência e a mortalidade, principalmente no primeiro dia de vida.

Almeida \& Mello Jorge 52 estudaram o retardo de crescimento intra-uterino - pequenos para idade gestacional (PIG) - como fator de risco para mortalidade neonatal (óbitos com menos de 28 dias) no Município de Santo André, São Paulo, usando o SINASC, o SIM e os registros hospitalares das maternidades. Para classificar os RN quanto ao CIUR, foi usada a curva de Lubchenco 30 . A idade gestacional baseou-se na DUM, com exclusão dos casos em que era desconhecida. A análise foi apenas bivariada, e encontrou-se um risco relativo de 8,3 para mortalidade neonatal, comparando PIG e não-PIG. Separando por idade gestacional, o risco relativo foi de 4,8 entre os prematuros e de 10,6 entre os RN a termo.

Por meio de estudo caso-controle, Aquino et al. 53 avaliaram os óbitos fetais com vinte semanas ou mais, ocorridos antes do trabalho de parto, em um hospital da periferia de São Paulo. Os controles eram os dois partos com nascidos vivos seqüenciais ao óbito fetal. Um questionário foi utilizado para levantar os fatores de risco, entre variáveis sócio-demográficas, reprodutivas e assistenciais. Após análise multivariada, somente as ocorrências de natimorto prévio, anemia, diabetes e hospitalização durante a gravidez foram identificadas como determinantes.

O trabalho de Andrade \& Szwarcwald 54 é um outro estudo ecológico, que explorou a relação entre indicadores sócio-econômicos (analfabetismo, moradia, renda) e maternos (idade, pré-natal, tipo de parto, tipo de hospital, coefi- ciente de natalidade) na distribuição geográfica da mortalidade neonatal do Município do Rio de Janeiro. Foi considerada a faixa etária de 0-3 dias, período em que se concentram os óbitos neonatais. As fontes de dados foram três sistemas: SINASC, SIM e o Censo Demográfico do IBGE. A análise estatística utilizou técnicas para dados de áreas e mediu a autocorrelação espacial. Os óbitos foram analisados globalmente, sem distinção por causa, ou IG e CIUR. As variáveis que melhor explicaram a concentração espacial de óbitos foram: proporção de mães adolescentes, proporção de residentes em favelas, proporção de chefes com renda igual ou inferior a um salário.

Almeida et al. 55 utilizaram os dados do SINASC e do SIM na cidade de São Paulo para avaliar a influência do peso, de fatores sóciodemográficos e assistenciais na mortalidade neonatal. Em um estudo caso-controle, analisaram os óbitos neonatais (menos de 28 dias) em conjunto e separadamente por faixa de peso (abaixo de 1.500g, 1.500-2.499 e igual ou superior a $2.500 \mathrm{~g}$ ), com exclusão das malformações congênitas e dos gemelares. A ausência de informação para algumas variáveis foi bem elevada: $30 \%$ para escolaridade, número de partos e natimorto prévio. No caso do pré-natal, esse percentual chegou a $60 \%$. Mesmo assim, os autores incluíram na análise todos os casos e controles, construindo uma categoria "sem informação". O modelo hierarquizado final para o conjunto apontou como determinantes: idade materna inferior a vinte anos, parto em unidade do SUS, baixo peso e prematuridade.

Andrade et al. 56 elegeram renda do chefe da família e escolaridade materna para estudar a relação entre desigualdades sócio-econômicas e óbito perinatal na cidade do Rio de Janeiro. A fonte de dados foi o inquérito 57 realizado na cidade entre 1999 a 2001, que descreveu os parâmetros populacionais das puérperas de 47 maternidades onde a mortalidade perinatal global foi $16 \%$. O estudo de Andrade não descreve as características dos óbitos, tais como peso, idade gestacional e causas. Utilizando as medidas de razão de taxas, risco atribuível, índice de dissimilaridade, coeficiente angular de desigualdade e índice de concentração (curva de Lorenz), os autores encontraram gradientes importantes de desigualdade sócio-econômica: quanto mais baixa a renda e/ou a escolaridade, maior a freqüência de óbitos perinatais.

c) Região Centro-Oeste: Morais Neto \& Barros 58 estudaram a mortalidade neonatal (menos de 28 dias) e pós-neonatal em Goiânia, acompanhando a coorte de nascimentos de 1992, usando dados do SINASC e do SIM. Atra- 
vés do procedimento de linkage dos bancos, foi explorada a relação entre grau de instrução materno, tipo de hospital, idade materna, peso ao nascer, categorias de IG, sexo, tipo de gravidez e de parto com a mortalidade. Não há referência ao método de estimação da IG constante das DNs em Goiânia. A análise foi por regressão logística, a partir de modelo hierárquico. Não foi utilizada classificação de causas de óbito. Baixo peso, prematuridade e nascimento em hospital público mostraram associação com mortalidade neonatal.

Em 2001, Morais Neto et al. 59 realizaram linkage dos bancos do SINASC e SIM para o período de 1992 a 1996, envolvendo 10 mil nascidos vivos em Goiânia, e definiram a distribuição espacial para mortalidade neonatal e pósneonatal. Para mortalidade neonatal, a área caracterizada como antiga periferia da cidade mostrou o maior risco, explicado possivelmente pelas deficiências na qualidade da atenção pré-natal, ao parto e neonatal. Foi utilizada a estratégia de análise de dados de área, adicionadas de taxas Bayesianas, para minimizar a instabilidade das probabilidades de morte. Não houve avaliação da mortalidade neonatal precoce em separado, nem análise por peso e idade gestacional.

d) Região Nordeste: Rouquayrol et al. 60 investigaram os fatores de risco (sócio-demográficos, reprodutivos, comportamentais e assistenciais) de natimortalidade em Fortaleza. Foram considerados os óbitos fetais com mais de vinte semanas de gestação, em uma unidade hospitalar de referência. Nesta unidade, a taxa de natimortalidade foi de $42,0 \%$. O desenho usado foi caso-controle: durante um ano (entre 1992 e 1993), foram captados 125 casos (natimortos) e 250 controles (nascidos vivos). Por razões logísticas, os casos não representaram o censo de natimortos do período. Os natimortos foram estudados de forma global, sem classificação pela causa básica ou distinção entre prematuridade e CIUR. Para análise estatística, foi utilizada regressão logística, a partir de um modelo hierárquico. Os determinantes foram: pré-natal inadequado (menos de quatro consultas), analfabetismo materno, renda menor que um salário-mínimo e morbidade materna. Adolescência, comparada à idade igual ou superior a vinte anos, teve efeito protetor.

\section{Discussão}

A mortalidade perinatal, apesar de decréscimo na última década, mostra-se ainda elevada no Brasil, com diferenças importantes no nível na- cional e regional. A natimortalidade variou de 9,0\%o em Belo Horizonte a 17,9\%o em Fortaleza, enquanto a neomortalidade precoce variou de 7,4\%o em Caxias do Sul a 15,3\%o em Fortaleza (Tabela 1). Observa-se a discordância entre a informação proveniente do SIH/SUS e a dos estudos baseados no SINASC/SIM e em dados primários, explicados por falhas na cobertura dos dois sistemas 20. Além dessas diferenças, os estudos de série temporal mostram mudanças profundas em pouco tempo, apontando a necessidade de constante monitoramento.

Os principais estudos sobre o tema foram desenvolvidos nas Regiões Sul e Sudeste e publicados em periódicos da área de saúde pública. Observou-se heterogeneidade quanto às definições adotadas, principalmente dos óbitos fetais, prejudicando a comparabilidade entre os estudos. Poucos trabalhos usaram classificações de óbito perinatal, sendo a de Wigglesworth 14, adaptada por Keeling 12, a mais citada. Predominou o uso de dados secundários; os grandes sistemas de informações têm sido cada vez mais utilizados, principalmente o SINASC e o SIM. Nem sempre os autores relataram a freqüência de dados ignorados nesses sistemas; em alguns casos, a ausência de informação sobre variáveis importantes como peso e idade gestacional foi superior a 30,0\%. A estratégia para lidar com essas perdas foi variada: alguns autores optaram pela exclusão dos casos sem informação, enquanto outros, pela imputação de valores compatíveis. Quanto à aferição das variáveis, a idade gestacional em geral é mensurada com base na DUM, mas alguns trabalhos não forneceram essa informação. Em muitos serviços de saúde, a declaração de nascido vivo, que alimenta o banco do SINASC, é preenchida por pediatras, os quais utilizam a avaliação de Capurro ou New Ballard para estimar IG. Estudo recente no Rio de Janeiro 61 mostrou que o New Ballard tendeu a superestimar a IG, gerando falsos negativos na classificação de prematuridade. Por outro lado, alguns estudos excluíram os casos com IG desconhecida, o que pode também enviesar os resultados. Nos poucos trabalhos que avaliaram os óbitos quanto ao crescimento intra-uterino, ainda se observou o uso da curva de Lubchenco, e apenas Menezes et al. 37 usaram a de Williams. Destaca-se também a mensuração do pré-natal, que foi apenas quantitativa na maioria dos estudos, com diferentes pontos de corte para número de consultas.

Existem mais estudos sobre a determinação da neomortalidade do que sobre natimortalidade e mortalidade perinatal. Os determinantes de mortalidade são estudados para os óbi- 
tos de forma global e não por causas específicas. Uma explicação possível é que, apesar das taxas elevadas, o número absoluto de óbitos é baixo, e a análise por subgrupos acarretaria perda de significância estatística, principalmente em estudos locais e períodos curtos. Entre os estudos de determinação, um foi transversal, quatro com o delineamento de coorte, quatro estudos caso-controle e três ecológicos, incorporando as técnicas de geoprocessamento. Notou-se a utilização crescente de análise estatística através de modelo hierárquico, tornando os resultados dos estudos mais consistentes.

Na maioria dos estudos, alguns fatores maternos mostraram-se associados à mortalidade fetal e neonatal, como idade elevada, presença de morbidade materna e história reprodutiva desfavorável, principalmente a ocorrência de natimorto prévio. Fatores proximais como sexo do RN, peso ao nascer, idade gestacional e baixo Apgar foram corroborados para mortalidade neonatal. Pré-natal inadequado, baixa renda e baixa escolaridade mostraram-se como fatores de risco para mortalidade tanto fetal como neonatal, embora alguns estudos apontassem valores não significativos. Outros determinantes tiveram resultados conflitantes ou não significativos, como adolescência, peso materno, tabagismo e alcoolismo. Isso pode ser explicado por diferenças regionais, mas principalmente por questões metodológicas, como instrumentos de coleta utilizados, os diferentes pontos de corte, a forma de registro destas variáveis nos bancos de dados e a impossibilidade de ajuste para fatores não incluídos nos sistemas de in-

\section{Resumo}

A mortalidade perinatal persiste como relevante evento em saúde pública, demandando estudos epidemiológicos, tanto para definir sua magnitude e variações temporais, como para identificar seus determinantes e apontar as intervenções adequadas. Existem ainda questões conceituais e metodológicas controversas, gerando heterogeneidade nos estudos e prováveis vieses. No Brasil, nos últimos anos, desponta uma produção crescente sobre o tema, principalmente no Sudeste e Sul. Foram revistos 24 artigos de 1996 a 2003, focalizando: definições e classificações utilizadas, fontes de dados, desenhos de estudo, formas de aferição das variáveis, modelos de análise estatística e principais resultados. A revisão mostrou a progressiva utilização de bancos de dados informatizados, principalmente $o$ SINASC e o SIM, o pequeno número de estudos sobre natimortalidade, a incorporação ainda incipiente das classificações de causas e a discordância em relação a alguns fatores de risco.

Mortalidade Perinatal; Literatura de Revisão; Métodos formação. Também pode ter contribuído o pequeno tamanho amostral de alguns estudos.

Conclui-se que a pesquisa sobre mortalidade perinatal pode ainda ser expandida e aprimorada no Brasil. O uso dos grandes sistemas de informação deve ser estimulado, permitindo o acompanhamento de grandes coortes de nascimentos; esforços devem ser feitos no sentido de melhorar a qualidade da informação e ampliar as possibilidades de investigação, acrescentando novas variáveis. Esquemas de classificação de causa, como o de Wigglesworth, poderiam ser incorporados tanto nos serviços de saúde, quanto na pesquisa. Quanto aos estudos de determinação, o número ainda pequeno e a heterogeneidade metodológica dificultam a sumarização dos resultados. Devem ser incentivados estudos primários, principalmente em nível regional, em virtude da grande diversidade nos eventos perinatais observada no Brasil. Se possível, deve-se realizar a investigação por subgrupos de causa específica, considerando-se principalmente a prematuridade e o crescimento intra-uterino, com adoção de curvas mais adequadas, como a de Williams 31 ou do Centro Latino-Americano de Perinatologia 32. Delineamentos como caso-controle e estudos ecológicos se mostram como ferramentas epidemiológicas interessantes para o estudo de fatores de risco. Finalizando, a aferição criteriosa das variáveis e a construção de modelos de determinação hierárquicos devem ser enfatizadas, garantindo a validade interna dos estudos e possibilitando análises mais refinadas.

\section{Colaboradores}

S. C. Fonseca contribuiu na busca bibliográfica, revisão e análise dos artigos e redação do texto. E. S. F. Coutinho colaborou na supervisão da análise dos artigos e redação do texto. 


\section{Referências}

1. Jackson DJ, Lang JM, Ganiats TG. Epidemiological issues in perinatal outcomes research. Paediatr Perinat Epidemiol 1999; 13:392-404.

2. World Health Organization. Perinatal mortality. A listing of available information. Geneva: World Health Organization; 1996.

3. Leal MC, Szwarcwald CL. Evolução da mortalidade neonatal no Estado do Rio de Janeiro, Brasil, de 1979 a 1993. 1 - Análise por grupo etário segundo região de residência. Rev Saúde Pública 1996; 30:403-12.

4. Hein HA, Lofgren MA. The changing pattern of neonatal mortality in a regionalized system of perinatal care: a current update. Pediatrics 1999; 104:1064-9.

5. Joseph KS, Kramer MS, Allen AC, Cyr M, Fair M, Ohlsson A, et al. Gestational age- and birthweightspecific declines in infant mortality in Canada, 1985-94. Fetal and Infant Health Study Group of the Canadian Perinatal Surveillance System. Paediatr Perinat Epidemiol 2000;14:332-9.

6. Cartlidge PHT, Stewart JH. Effect of changing the stillbirth definition on evaluation of perinatal mortality rates. Lancet 1995; 346:4486-8.

7. Gourbin C, Masuy-Stroobant G. Registration of vital data: are live births and stillbirths comparable all over Europe? Bull World Health Organ 1995; 73:449-60.

8. Richardus JH, Graafmans WC, van der Pal-de Bruin KM, Amelink-Verburg MP, Verloove-Vanhorick SP, MacKenbach JP. An European concerted action investigating the validity of perinatal mortality as an outcome indicator for the quality of antenatal and perinatal care. J Perinat Med 1997; 25:313-24.

9. Conde-Agudelo A, Belizan JM, Diaz-Rossello JL. Epidemiology of fetal death in Latin America. Acta Obstet Gynecol Scand 2000; 79:371-8.

10. De Lorenzi DRS, Tanaka ACA, Bozzetti MC, Ribas FE, Weissheimer L. A natimortalidade como indicador de saúde perinatal. Cad Saúde Pública 2001; 17:141-6.

11. Kramer MS, Liu S, Luo Z, Yuan H, Platt RW, Joseph KS. Fetal and Infant Health Study Group of the Canadian Perinatal Surveillance System. Analysis of perinatal mortality and its components: time for a change? Am J Epidemiol 2002; 156:493-7.

12. Keeling JW, MacGillivray I, Golding J, Wigglesworth J, Berry J, Dunn PM. Classification of perinatal death. Arch Dis Child 1989; 64:1345-51.

13. Winbo I, Serenius F, Dahlquist G, Kallen B. NICE, a new cause of death classification for stillbirths and neonatal deaths. Int J Epidemiol 1998; 27: 499-504.

14. Wigglesworth JS. Monitoring perinatal mortality - a pathophysiological approach. Lancet 1980; 2:684-6.

15. Fundação Sistema Estadual de Dados. Mortalidade infantil e desenvolvimento. Conjuntura Demográfica 1991; 14/15:49-50.

16. Lansky S, França E, Leal MC. Mortalidade perinatal e evitabilidade: revisão da literatura. Rev Saúde Pública 2002; 36:759-72.

17. Almeida MF, Mello-Jorge MHP. O uso da técnica de linkage de sistemas de informação em estudos de coorte sobre mortalidade neonatal. Rev Saúde Pública 1996; 30:141-7.
18. Carvalho ML, Silver L. Confiabilidade da declaração da causa básica de óbitos neonatais: implicações para o estudo da mortalidade prevenível. Rev Saúde Pública 1995; 29:342-8.

19. Noronha CP, Silva RI, Theme MM, Monteiro EA. Concordância dos dados entre os sistemas de informação sobre nascidos vivos e de mortalidade para os óbitos neonatais da cidade do Rio de Janeiro. Anais do Congresso de Epidemiologia; 1998 Agosto 2-7; Rio de Janeiro, Brasil. Rio de Janeiro: ABRASCO; 1998.

20. Schramm JMA, Szwarcwald CL. Sistema hospitalar como fonte de informações para estimar a mortalidade neonatal e a natimortalidade. Rev Saúde Pública 2000; 34:272-9.

21. Rodrigues L, Kirkwood BR. Case-control designs in the study of common diseases: updates on the demise of the rare disease assumption and the choice of sampling scheme for controls. Int J Epidemiol 1990; 19:205-13.

22. Barros FC. Fatores de risco de natimortalidade em Fortaleza: um estudo de caso-controle. J Pediatr (Rio J) 1996; 72:361-2.

23. Forssas E, Gissler M, Sihvonen M, Hemminki E. Maternal predictors of perinatal mortality: the role of birthweight. Int J Epidemiol 1999; 28:475-8.

24. Kramer MS, Séguin L, Lydon J, Goulet L. Socioeconomic disparities in pregnancy outcome: why do the poor fare so poorly? Paediatr Perinat Epidemiol 2000; 14:194-210.

25. Kramer MS, Goulet L, Lydon J, Séguin L, MacNamara H, Dassa C, et al. Socio-economic disparities in preterm birth: causal pathways and mechanisms. Paediatr Perinat Epidemiol 2001; 15 Suppl 2:104-23.

26. Victora CG, Huttly SR, Fuchs SC, Olinto MT. The role of conceptual frameworks in epidemiological analysis: a hierarquical approach. Int J Epidemiol 1997; 26:224-7.

27. Capurro H, Konichezky S, Fonseca D, CaldeyroBarcia R. A simplified method for diagnosis of gestational age in the newborn infant. J Pediatr 1978; 93:120-2.

28. Ballard JL, Khoury JC, Wedig K, Wang L, EilersWalsman BL, Lipp R. New Ballard score, expanded to include extremely premature infants. J Pediatr 1991; 119:417-23.

29. Alexander GR, Tompkins ME, Petersen DJ, Hulsey TC, Mor J. Discordance between LMP-based and clinically estimated gestational age: implications for research, programs and policy. Public Health Rep 1995; 110:395-402.

30. Lubchenco LO, Hansman C, Dressler M, Boyd E. Intrauterine growth as estimated from liveborn birth-weight data at 24 to 42 weeks of gestation. Pediatrics 1963; 32:793-800.

31. Williams RL, Creasy RK, Cunningham GC, Hawes WE, Norris FD, Tashiro M. Fetal growth and perinatal viability in California. Obstet Gynecol 1982; 59:624-32.

32. Centro Latino-americano de Perinatología y Desarollo Humano. Vigilancia del crecimiento fetal. Manual de autoinstrucción. Montevideo: Centro Latino-americano de Perinatología y Desarollo Human, Organización Panamericana de la Salud/ 
Organización Mundial de la Salud; 1992. (Publicación Científica 1261).

33. Margotto PR. Curvas de crescimento intra-uterino: estudo de 4413 recém-nascidos únicos de gestações normais. J Pediatr (Rio J) 1995; 71:11-21.

34. Alexander GR, Himes JH, Kaufman RB, Mor J, Kogan M. A United States national reference for fetal growth. Obstet Gynecol 1996; 87:163-8.

35. Marsal K, Persson PH, Larsen T, Lilja H, Selbing A, Sultan B. Intrauterine growth curves based on ultrasonically estimated foetal weights. Acta Paediatr 1996; 85:843-8.

36. Kramer MS, Platt RW, Wen SW, Joseph KS, Allen A, Abrahamowicz M, et al. A new and improved population-based Canadian reference for birth weight for gestational age. Pediatrics 2001; 108:E35.

37. Menezes AMB, Barros FC, Victora CG, Alves C, Rocha C, Albernaz E, et al. Mortalidade perinatal em duas coortes de base populacional no Sul do Brasil: tendências e diferenciais. Cad Saúde Pública 1996; 12 Suppl 1:33-41.

38. Miura E, Faillace LH, Fiori H. Mortalidade perinatal e neonatal no Hospital de Clínicas de Porto Alegre. AMB Rev Assoc Med Bras 1997; 43:35-9.

39. De Lorenzi DRS, Tanaka ACA, Ribas FE, Rech FS, Weissheimer LA. Perfil epidemiológico da natimortalidade em Caxias do Sul. Rev Cient AMECS 1999; 829-33.

40. Leal MC, Szwarcwald CL. Evolução da mortalidade neonatal no Estado do Rio de Janeiro, Brasil (1979 a 1993): análise por causa segundo grupo de idade e região de residência. Cad Saúde Pública 1996; 12:243-52

41. Sardas R, Manço ARX. Perdas fetais e natimortalidade no Município de Ribeirão Preto-SP, Brasil, 1991 e 1992. Medicina (Ribeirão Preto) 1997; 30:508-13.

42. Organização Mundial da Saúde. Manual da Classificação Estatística Internacional de Doenças, Lesões e Causas de Óbitos - Nona Conferência de Revisão. São Paulo: Centro Brasileiro de Classificação de Doenças em Português; 1985.

43. Aquino MMA, Cecatti JG. Epidemiologia do óbito fetal em população de baixa renda. Rev Bras Ginecol Obstet 1998; 20:71-5.

44. Mariotoni GGB, Barros Filho AA. Peso ao nascer e mortalidade hospitalar entre nascidos vivos, 19751996. Rev Saúde Pública 2000; 34:71-6.

45. Lansky S, França E, Leal MC. Mortes perinatais evitáveis em Belo Horizonte, Minas Gerais, 1999. Cad Saúde Pública 2002; 18:1389-400.

46. Leite AJM, Marcopito LF, Diniz RLP, Silva AVS, Souza LCB, Borges JC, et al. Mortes perinatais no Município de Fortaleza, Ceará: o quanto é possível evitar? J Pediatr (Rio J) 1997; 73:388-94.

47. Ribeiro VS, Silva AAM. Tendências da mortalidade neonatal em São Luís, Maranhão, Brasil, 1979 a 1996. Cad Saúde Pública 2000; 16:429-38.

48. Menezes AMB, Barros FC, Victora CG, Tomasi E, Halpern R, Oliveira ALB. Fatores de risco para mortalidade perinatal em Pelotas, RS, 1993. Rev Saúde Pública 1998; 32:209-16.

49. Araújo BF, Bozzetti MC, Tanaka ACA. Mortalidade neonatal precoce no Município de Caxias do Sul: um estudo de coorte. J Pediatr (Rio J) 2000; 76: 200-6.
50. Vardanega K, De Lorenzi DRS, Spiandorello WP, Zapparoli MF. Fatores de risco para natimortalidade em um hospital universitário da Região Sul do Brasil. Rev Bras Ginecol Obstet 2002; 24:617-22.

51. Leal MC, Szwarcwald CL. Características da mortalidade neonatal no Estado do Rio de Janeiro na década de 80: uma visão espaço-temporal. Rev Saúde Pública 1997; 31:457-65.

52. Almeida MF, Mello-Jorge MHP. Pequenos para idade gestacional: fator de risco para mortalidade neonatal. Rev Saúde Pública 1998; 32:217-24.

53. Aquino MMA, Cecatti JG, Mariani Neto C. Risk factors associated to fetal death. São Paulo Med J 1998; 116:1852-7.

54. Andrade CLT, Szwarcwald CL. Análise espacial da mortalidade neonatal precoce no Município do Rio de Janeiro, 1995-1996. Cad Saúde Pública 2001; 17:1199-210.

55. Almeida MF, Novaes HMD, Alencar GP, Rodrigues LC. Mortalidade neonatal no Município de São Paulo: influência do peso ao nascer e fatores sócio-demográficos e assistenciais. Rev Bras Epidemiol 2002; 5:93-107.

56. Andrade CLT, Szwarcwald CL, Gama SGN, Leal MC. Desigualdades sócio-econômicas do baixo peso ao nascer e da mortalidade perinatal no $\mathrm{Mu}-$ nicípio do Rio de Janeiro, 2001. Cad Saúde Pública 2004; 20 Suppl 1:S44-51.

57. Leal MC, Gama SGN, Campos MR, Cavalini LT, Garbayo LS, Brasil CLP, et al. Fatores associados à morbi-mortalidade perinatal em uma amostra de maternidades públicas e privadas do Município do Rio de Janeiro, 1999-2001. Cad Saúde Pública 2004; 20 Suppl. 1:S20-33.

58. Morais Neto OL, Barros MBA. Fatores de risco para mortalidade neonatal e pós-neonatal na Região Centro-Oeste do Brasil: linkage entre bancos de dados de nascidos vivos e óbitos infantis. Cad Saúde Pública 2000; 16:477-85.

59. Morais Neto OL, Barros MBA, Martelli CMT, Silva AS, Siqueira Jr. JB. Diferenças no padrão de ocorrência da mortalidade neonatal e pós-neonatal no Município de Goiânia, Brasil, 1992-1996: análise espacial para identificação das áreas de risco. Cad Saúde Pública 2001; 17:1241-50.

60. Rouquayrol MZ, Correia LL, Barbosa LMM, Xavier LGM, Oliveira JW, Fonseca W. Fatores de risco de natimortalidade em Fortaleza: um estudo caso-controle. J Pediatr (Rio J) 1996; 72:374-8.

61. Moraes CL, Reichenheim ME. Validade do exame clínico do recém-nascido para a estimação da idade gestacional: uma comparação do escore New Ballard com a data da última menstruação e ultra-sonografia. Cad Saúde Pública 2000; 16:83-94.

62. Schramm JMA, Szwarcwald CL. Diferenciais nas taxas de mortalidade neonatal e natimortalidade hospitalares no Brasil: um estudo com base no Sistema de Informações Hospitalares do Sistema Único de Saúde (SIH/SUS). Cad Saúde Pública 2000; 16:1031-40.

Recebido em 24/Jun/2003

Versão final reapresentada em 26/Nov/2003

Aprovado em 20/Fev/2004 